\title{
Human Placenta-Derived ECM Supports Tri-Lineage Differentiation of Human Induced Pluripotent Stem Cells
}

\author{
Angela C. Murchison*, Justin J. Odanga*, Michelle L. Treadwell, \\ Erick K. Breathwaite, Jessica R. Weaver, Jung Bok Lee \\ Institute of Regenerative Medicine, LifeNet Health, Virginia Beach, VA, USA
}

\begin{abstract}
Human pluripotent stem cells (hPSCs) hold great promise for future applications in drug discovery and cell therapies. hPSC culture protocols require specific substrates and medium supplements to support cell expansion and lineage specific differentiation. The animal origin of these substrates is a severe limitation when considering the translation of hPSC derivatives to the clinic and in vitro disease modeling. The present study evaluates the use of a human placenta-derived extracellular matrix (ECM) hydrogel, HuGentra ${ }^{\mathbb{B}}$, to support tri-lineage differentiation of human induced pluripotent stem cells (hiPSCs). Lineage-specific embryoid bodies (EBs) were plated onto three separate matrices, and differentiation efficiency was evaluated based on morphology, protein, and gene expression. HuGentra was found to support the differentiation of hiPSCs to all three germ layers: ectodermal, mesodermal, and endodermal lineages. hiPSCs differentiated into neurons, cardiomyocytes, and hepatocytes on HuGentra had similar morphology, protein, and gene expression compared to differentiation on Matrigel or other cell preferred matrices. HuGentra can be considered as a suitable human substrate for hiPSC differentiation.
\end{abstract}

Keywords: hiPSC, Tri-lineage, Differentiation, Human ECM

\section{Introduction}

Differentiated cell populations from hiPSCs requires highly optimized extracellular matrices and growth factors. Matrigel is a frequently used extracellular matrix (ECM) for differentiation and support of hiPSCs. It is able to provide high biologic activity due to its complex combination

Received: May 1, 2020, Revised: June 5, 2020,

Accepted: June 11, 2020, Published online: August 31, 2020

Correspondence to Jung Bok Lee

Institute of Regenerative Medicine, LifeNet Health, 1864 Concert

Drive, Virginia Beach, VA 23453, USA

Tel: +1-757-609-4376, Fax: +1-757-609-4191

E-mail: jungbok_lee@lifenethealth.org

*These authors contributed equally to this work.

(a) This is an open-access article distributed under the terms of the Creative Commons Attribution Non-Commercial License (http://creativecommons.org/ licenses/by-nc/4.0/), which permits unrestricted non-commercial use, distribution, and reproduction in any medium, provided the original work is properly cited.

Copyright (C) 2020 by the Korean Society for Stem Cell Research of laminin, collagen type IV, proteoglycans, and growth factors including transforming growth factor- $\beta$ (TGF- $\beta$ ), fibroblast growth factor (FGF), epidermal growth factor (EGF), platelet-derived growth factor (PDGF), and insulin-like growth factor (IGF) (1).

However, the disease and animal origin of Matrigel, creates concerns and complications when considering clinical applications of hiPSC-derived cells. Purified proteins such as gelatin and rat tail collagen are also used as ECMs for lineage and stage specific hiPSC differentiations (2-7). Similarly, animal origin remains a concern. Because of this regulatory hurdle, a human matrix is highly desirable for the production of hiPSC-derived cell populations.

Human ECMs have been demonstrated to be successful for the production of hiPSC-derived neural cells (8) and hepatocytes (9) individually. It has also been shown that human ECMs can induce tissue-specific differentiation of hiPSCs (10); however, no humanized matrix has been shown to support the differentiation of hiPSCs into all three lineages. hiPSC-derived cells originating from the 
same supporting material may be primed to work in synchrony for in vivo and in vitro applications.

We have produced an ECM derived from human placentas, HuGentra. This matrix is rich in laminins, collagens, fibronectin, glycoproteins, and stem cell-recruiting growth factors such as vascular endothelial growth factor (VEGF), hepatocyte growth factor (HGF), FGF, PDGF, IGF, and EGF (11). HuGentra has also been shown in vitro to support the proliferation and function of adipose derived stem cells and a commercially available hiPSC-derived cardiomyocyte cell line. In vivo it has been shown to have regenerative capacity as well (11). In this current study, HuGentra's ability to support hiPSC differentiation into cell types of the three lineages is compared to Matrigel and lineage preferred matrices.

\section{Materials and Methods}

\section{hiPSC maintenance}

hiPSCs were maintained in feeder-free conditions on Matrigel with MEF conditioned medium. MEF conditioned medium was prepared by incubating complete medium DMEM/F12 supplemented with 20\% KnockOut Serum Replacement (KSR), 1 mM MEM Non-Essential Amino Acid Solution (NEAA), and $2 \mathrm{mM}$ L-Glutamine with irradiated CF1 mouse embryonic fibroblasts (Thermo Fisher Scientific, Waltham, MA). Media was collected and filter sterilized. MEF conditioned medium was supplemented with $40 \mathrm{ng} / \mathrm{ml}$ of recombinant human basic fibroblast growth factor (Rh-bFGF) (Thermo). hiPSC lines were generated from adult dermal fibroblasts and neonatal foreskin fibroblasts (Lifenet Health) according to manufacturer's instructions using the Stemfect RNA Transfection Kit (StemGent, Cambridge, MA) and from osteoblasts (Lifenet Health) using the CytoTune-iPS Sendai Reprogramming Kit (Thermo). hiPSCs were fed daily and passaged weekly using collagenase type IV (Thermo) in combination with mechanical scraping.

\section{Vessel coating}

Matrigel (Corning, Corning New York) was diluted $1: 30$ with cold DMEM/F12. Gelatin (Thermo) came in a ready to use $0.1 \%$ solution. Rat Tail Collagen 1 (Thermo) was prepared according to the manufacturer's instructions. HuGentra was placed on ice at $4{ }^{\circ} \mathrm{C}$ until fully thawed, and a dilution of approximately $0.165 \mathrm{mg} / \mathrm{ml}$ was prepared in cold DMEM/F12. All matrices were incubated at $4{ }^{\circ} \mathrm{C}$ overnight prior to use.

\section{hiPSC differentiation towards neural cells}

Prior to the neural differentiation, hiPSCs were cultured with mTeSR1 (STEMCELL Technologies, Vancouver, Canada) on Matrigel for at least 2 passages as a pre-commitment process (4). EBs were then formed by transferring the resulting hiPSC aggregates to a 6-well ultra-low attachment (ULA) plate (Corning) at a $1: 1$ seeding ratio. Cells were cultured in MEF conditioned medium supplemented with $1 \times \mathrm{B}-27$ and $8 \mathrm{ng} / \mathrm{ml} \mathrm{Rh-bFGF} \mathrm{(Thermo).}$ After a week in suspension, the formed EBs were transferred onto either Matrigel, gelatin, or HuGentra coated plates.

\section{hiPSC differentiation towards cardiomyocytes}

hiPSCs were cultured on Matrigel for one passage prior to starting the cardiomyocyte differentiation. EBs were formed as described above. The EBs were cultured for 7 days in MEF conditioned medium supplemented with $10 \mathrm{ng} / \mathrm{ml}$ BMP4 (Thermo), 10\% FBS (Atlanta Biologicals, Flowery Branch, GA), $82 \mu \mathrm{g} / \mathrm{ml}$ ascorbic-2-phosphate (Sigma-Aldrich, St. Louis, MO), $10 \mathrm{ng} / \mathrm{ml}$ Activin A, and $25 \mathrm{ng} / \mathrm{ml}$ Wnt3a (R\&D Systems, Minneapolis, MN). The EBs were then transferred onto either Matrigel, gelatin, or HuGentra coated plates.

\section{hiPSC differentiation towards hepatocytes}

hiPSCs were cultured on Matrigel for one passage prior to starting the hepatocyte differentiation. EBs were formed as described above. The EBs were cultured for 7 days in MEF conditioned medium supplemented with $10 \% \mathrm{FBS}$ and $100 \mathrm{ng} / \mathrm{ml}$ Activin A. The EBs were then transferred onto either Matrigel, Collagen 1, or HuGentra coated plates and cultured with hepatocyte medium containing DMEM/F12, $1 \mathrm{mM}$ NEAA, 1\% Glutamax (Thermo), $10 \% \mathrm{FBS}$, and $100 \mathrm{ng} / \mathrm{ml}$ Activin A. Seven days after EB attachment to the matrices $100 \mathrm{nM}$ dexamethasone, $20 \mathrm{ng} / \mathrm{mL}$ Oncostatin M (Sigma-Aldrich), and $20 \mathrm{ng} / \mathrm{mL}$ HGF (R\&D Systems) were added to the media.

\section{Immunocytochemical analysis}

hiPSCs were live-stained using StainAlive Tra-1-81 antibody (Stemgent, $1: 100$ ) for one hour at $37^{\circ} \mathrm{C}$. hiPSCs were rinsed once with $1 \times$ DPBS $-\mathrm{Ca},-\mathrm{Mg}$ (Thermo), and imaged in a DMEM-phenol free media (Thermo). Differentiated cells were prepared according to manufacturer instructions using the Fixation and Permeabilization Solution Kit (BD BioSciences, San Jose, CA). Primary antibodies were applied at $1: 100$ dilution overnight at $4^{\circ} \mathrm{C}$ : Beta-III Tubulin (Tuj-1, R\&D Systems), Cardiac Troponin$\mathrm{T}$ (cTnT, Abcam Cambridge, MA), Albumin (ALB, Abcam), 
and alpha fetal protien (AFP, Abcam). Secondary antibodies (Thermo; $1: 1,000$ ) were applied at $4^{\circ} \mathrm{C}$ for 1 hour. Cells were counterstained with DAPI (Vector Labs, Burlingame, CA; $1 \mathrm{ug} / \mathrm{ml})$. Images were acquired on an EVOS FLc (Thermo). Images were processed and analyzed in Image J Ver. 1.52A.

\section{Flow cytometry analysis}

Cells were fixed for one hour at $4{ }^{\circ} \mathrm{C}$ using a Fixation and Permeabilization Solution Kit (BD). Primary antibodies were applied to fixed cells at 1:200 dilution: Tuj-1, A2B5, cTnT, nestin (NES, Abcam), AFP, hepatocyte nuclear factor 4 (HNF4, Abcam), and ALB. Secondary antibodies were applied at $4{ }^{\circ} \mathrm{C}$ for 1 hour. Samples were run on a C6 flow cytometer (BD) and analyzed with CFlow Plus Software Ver. 1.0.227.4 (BD).

\section{Gene expression}

RNA was isolated from cells lysed with TRIzol (Thermo) and purified with the RNeasy mini kit (Qiagen, Germantown, MD). cDNA was synthesized using the PrimeScript RT reagent Kit (Takara Bio, Shiga, Japan) according to the manufacturer's instructions. All qRT-PCR amplifications were carried out in $20 \mu 1$ reactions using the QuantiNova SYBR Green RT-PCR kit (Qiagen) according to the manufacturer's instructions. The following custom primer sequences (5'-3') were used: OLIG2 (Forward TGGCTTCAAGTCATCCTCGTC, Reverse ATG GCGATGTTGAGGTCGTG), HNF4A (Forward CGAAGG TCAAGCTATGAGGACA, Reverse ATCTGCGATGCTG GCAATCT), ALB (Forward- TGCAACTCTTCGTGAAA CCTATG, Reverse ACATCAACCTCTGGTCTCACC), TNNT2 (Forward GGAGGAGTCCAAACCAAAGCC, Reverse TCAAAGTCCACTCTCTCTCCATC), and GAPDH (Forward GGTCACCAGGGCTGCTTTTA, Reverse -GGATCTCGC TCCTGGAAGATG). Data was normalized to the housekeeping gene GAPDH. All reactions were carried out using the StepOnePlus Real-Time PCR System (Thermo). Data was analyzed using StepOneTM Software Ver. 2.3 (Thermo) and the $2^{-\Delta \Delta \mathrm{C}}$ method.

\section{Quantifying differentiated cardiomyocyte and hepatocyte colonies}

Attached EBs were counted one day post plating on Matrigel, Rat Tail Collagen I, or HuGentra. The number of EBs initially attached and EBs showing contractile
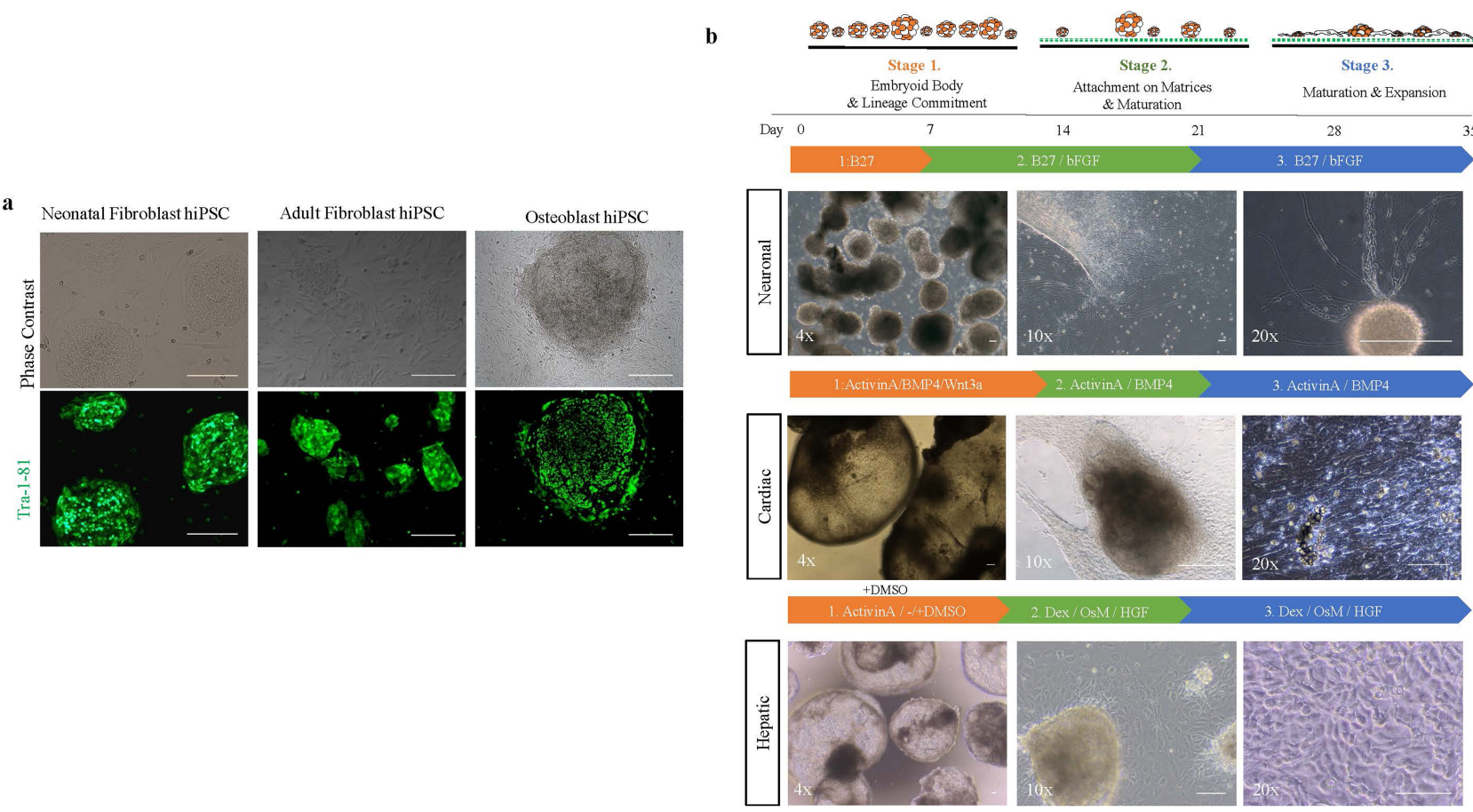

Fig. 1. Characterization of hiPSC lines and differentiation schematic. (a) Three hiPSC lines derived from adult dermal fibroblasts, neonatal foreskin fibroblasts, and adult human osteoblasts display the typical hiPSC morphology and stain for Tra-1-81. Representative images were taken $10 \times$. (b) Each stage of differentiation is displayed with the respective media supplements used to induce that stage of the differentiation as indicated by the numbering. Representative images are shown of the three lineages during each stage of the differentiations. Representative images were taken $4 \times, 10 \times$, and $20 \times$. 
beating was recorded on day 35 of differentiation. Similarly, for the hepatocyte differentiation, the number of attached EBs and the number of attached EB colonies having characteristic cuboidal hepatocyte morphology, nucleus to cytoplasm ratio, and clear boundaries were recorded on day 35 of differentiation.

\section{Statistical analysis}

Statistical significance was determined using a one way ANOVA with a post-hoc Tukey test for all quantitative data sets with $95 \%$ confidence and $\mathrm{p}<0.05$. All quantitative analysis was performed with 3 biologic replicates. All error bars denote standard deviation.

\section{Results and Discussion}

\section{Generation of hiPSC-induced neurons, cardiomyoctes, and hepatocytes}

hiPSCs were derived from multiple types of primary human cells including neonatal foreskin fibroblasts, adult dermal fibroblasts, and adult osteoblasts. Prior to mul- ti-lineage differentiation, hiPSCs were found to have characteristic hiPSC colony formation with clear boundaries and small, round, single cells containing multiple nucleoli. To confirm morphology, ICC for undifferentiated pluripotent human stem cells was performed using Tra-1-81 (Fig. 1a). As expected, hiPSCs batches showed clear expression of Tra-1-81. hiPSCs were differentiated in three stages to achieve target lineage (Fig. 1b). hiPSCs first were committed to a specific lineage during the formation of EBs. During stage two, attachment, outgrowth, and maturation of EBs on various matrices occurred. Cells matured and expanded during stage three to complete differentiation to target lineage.

\section{hiPSC differentiation towards neurons}

Prior to plating EBs on substrates, the efficiency of the ectodermal priming completed in stage one was assessed by expression of the neural progenitor marker A2B5 by flow cytometry. Primed EBs expressed A2B5 at a 12\% $2.3 \%$ efficiency (Fig. 2a). Primed cells were then plated onto gelatin, Matrigel, or HuGentra for the maturation and ex-
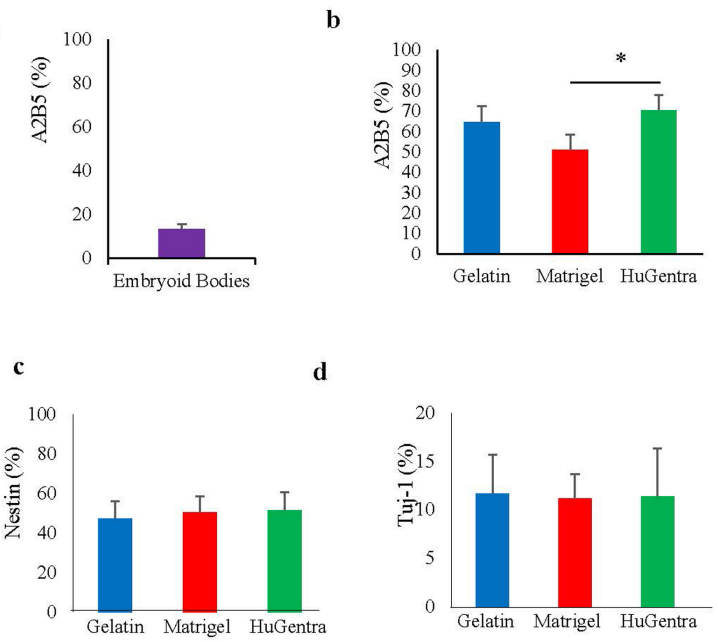

e
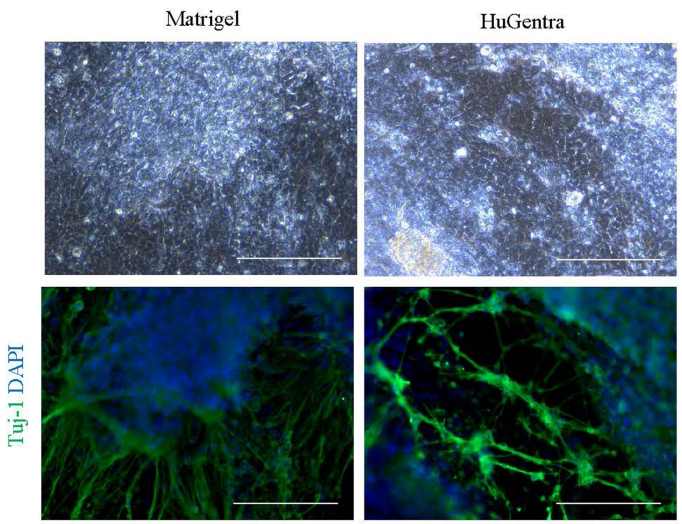

f

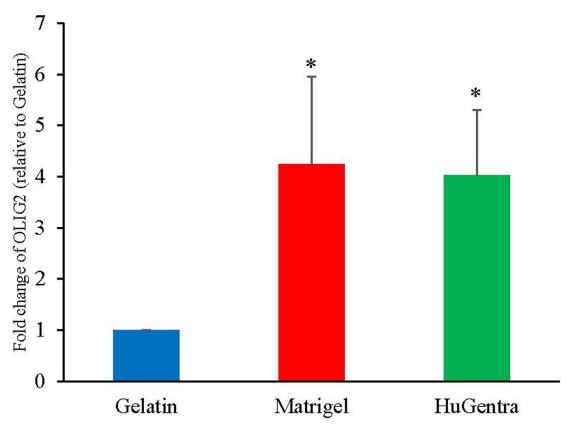

Fig. 2. Characterization of hiPSC-derived neurons on matrices. (a) The ectodermal lineage marker A2B5 was expressed at a frequency of $12 \% \pm 2.3 \%$ by dissociated EBs. (b) A2B5 expression following differentiation on gelatin, Matrigel, or HuGentra. ${ }^{*} \mathrm{p}<0.05$ to hiPSCs differentiated on HuGentra versus Matrigel. Flow analysis for neuronal markers. (c) NES and (d) Tuj-1. (e) ICC of Tuj-1 and representative morphological images. Representative images were taken $20 \times$. (f) The gene expression of OLIG2 in Matrigel and HuGentra normalized to expression on gelatin. Error bars represent standard deviation. ${ }^{*} \mathrm{p}<0.05$ to hiPSCs differentiated on gelatin. $\mathrm{n} \geq 3$. 
pansion of the hiPSCs into neural cells. At the completion of the differentiation, hiPSCs on HuGentra $(70.6 \% \pm 7.8 \%)$ had significantly more A2B5 positive cells than the hiPSCs differentiated on the Matrigel $(51.0 \% \pm 7.5 \%)\left({ }^{*} \mathrm{p}<0.05\right)$. Gelatin $(64.8 \% \pm 7.6 \%)$ showed increased expression compared to Matrigel; however, it was not significant (Fig. 2b). The neural markers NES (Fig. 2c) and Tuj-1 (Fig. 2d) were expressed at similar levels on all substrates with no significant differences. ICC of Tuj-1 showed characteristic microtubule staining along the neuron (Fig. 2e). Expression of the neuroectodermal progenitor marker oligodendrocyte transcription factor 2 (OLIG2) was significantly increased in cells differentiated on Matrigel and HuGentra compared to gelatin with a $4.2 \pm 1.7$ and $4.0 \pm 1.3$ fold change respectively $\left({ }^{*} \mathrm{p}<0.05\right)$ (Fig. $\left.2 \mathrm{f}\right)$.

\section{hiPSC differentiation towards cardiomyocytes}

EBs primed towards the mesoderm lineage were plated onto gelatin, Matrigel, or HuGentra for the maturation and expansion of cardiomyocytes (Fig. 3). Proliferating colonies on all matrices began to contract by day 14 (see arrows) of differentiation start (Fig. 3a). The percentage of beating colonies on gelatin $(22.1 \pm 10.8)$, Matrigel $(19.4 \pm$ $6.8)$, and HuGentra $(22.9 \pm 7.9)$ showed no significant differences (Fig. 3b). To determine the efficiency of cardiomyocyte differentiation, the mature cardiac marker, cTnT was analyzed by flow cytometry (Fig. 3c). Expression of cTnT on gelatin $(54.4 \% \pm 3.8 \%)$, Matrigel $(62.0 \% \pm$ $4.2 \%)$, and HuGentra $(57.9 \% \pm 3.9 \%)$ showed no significant differences. Further analysis of the cell population by ICC indicated the characteristic striated pattern of cTnT on all three matrices (Fig. 3d). Expression of the gene encoding cTnT, TNNT2, was analyzed (Fig. 3e). No significant differences in TNNT2 expression was seen between HuGentra and Matrigel compared to gelatin with a $1.2 \pm 0.11$ and $0.85 \pm 0.19$ fold change respectively.

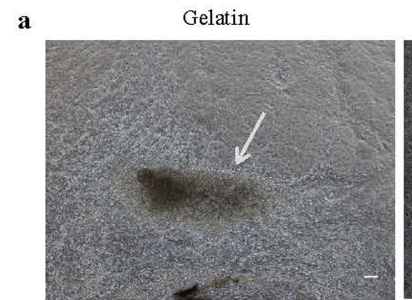

c

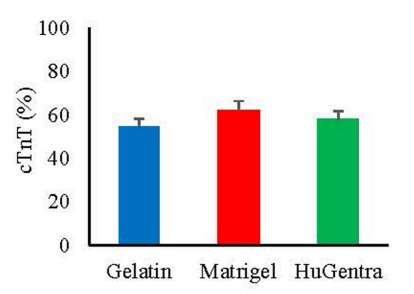

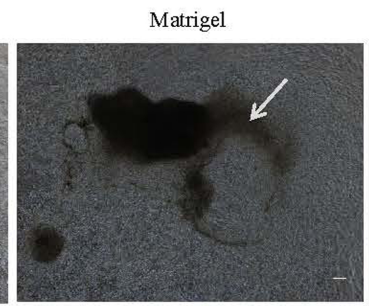

d

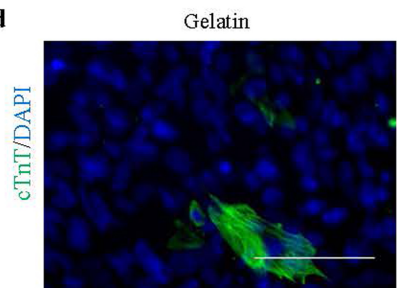

HuGentra

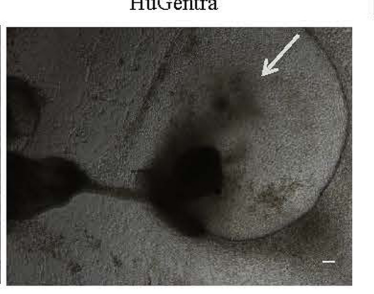

Matrigel
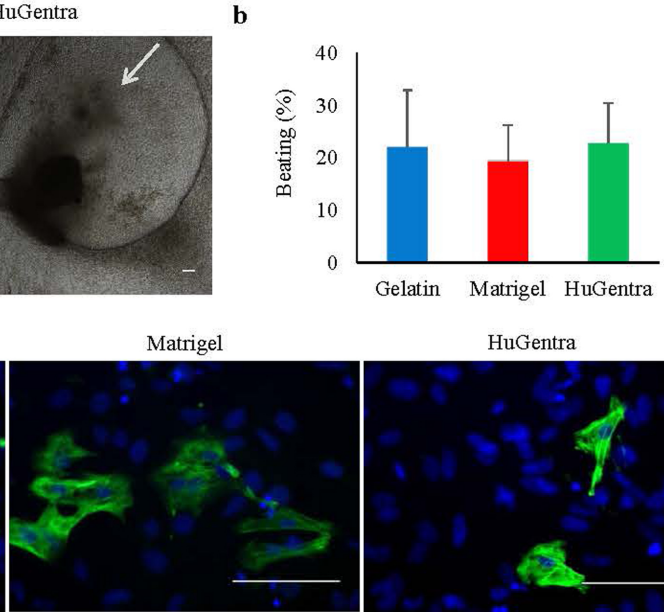

HuGentra

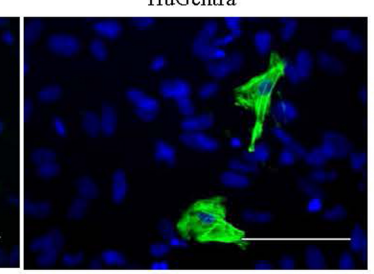

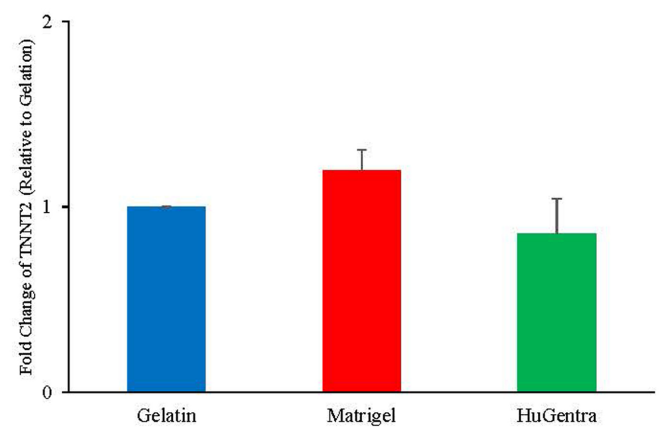

Fig. 3. Characterization of hiPSC-derived cardiomyocytes on matrices. (a) Following 14 days of differentiation, contracting colonies were found on all matrices as indicated by arrows. Representative images were taken $4 \times$. (b) No significant differences in the frequency of the contracting colonies relative to the total attached colonies when quantitated on day 30. (c) After dissociation, the cells were assessed for expression cTnT via flow cytometry. The level of cTnT was similar between all three matrices. (d) cTnT expression was confirmed with ICC on the three matrices. Representative images were taken $20 \times$. (e) Relative gene expression of the mature cardiac marker, TNNT2, was also shown and normalized to expression of gelatin. Error bars represent standard deviation. $\mathrm{n} \geq 3$. 


\section{hiPSC differentiation towards hepatocytes}

EBs primed towards the endoderm lineage were plated onto Collagen I, Matrigel, or HuGentra for the maturation and expansion of hepatocytes (Fig. 4). Characteristic hepatic morphology, polygonal shapes with large round nuclear envelopes, and a large nucleus to cytoplasm ratio were observed on all matrices (Fig. 4a). Following attachment, EBs were identified and counted on each matrix (Fig. 4b). The quantity of hepatic like colonies present surrounding each attached $\mathrm{EB}$ was quantitated on day 35 of the differentiation. Collagen I $(68 \% \pm 12 \%)$, Matrigel $(60 \% \pm 23 \%)$, and HuGentra $(75 \% \pm 25 \%)$ showed no significant differences of hepatic colony formation (Fig. 4c). Flow cytometry marker expression of ALB (Matrigel 79.9\% 2.9\%, Collagen $180.5 \% \pm 2.1 \%$, HuGentra $82.3 \% \pm 3.9 \%$ ), HNF4 (Matrigel 38.6\% $\pm 11.3 \%$, Collagen $135.2 \% \pm 7.2 \%$, HuGentra $37.9 \% \pm 10.3 \%$ ), and AFP (Matrigel $22.7 \% \pm 2 \%$, Collagen $123.1 \% \pm 11.6 \%$, HuGentra $22.9 \% \pm 6.4 \%$ ) was performed. No significant differences were seen (Fig. 4d).
The presence of ALB and AFP were further verified by ICC (Fig. 4e). No visible differences of expression were seen between the matrices. HNF4 and $A L B$ levels were further substantiated by the relative gene expression of the cells differentiated on Collagen 1 and HuGentra relative to the expression level on Matrigel (Fig. 4f). HNF4 expression was significantly higher in cells differentiated on HuGentra and Collagen 1 compared to cells differentiated on Matrigel. $A L B$ expression was significantly higher in cells differentiated on Collagen 1 compared to cells differentiated on Matrigel $\left({ }^{*} \mathrm{p}<0.05\right)$.

In conclusion, the present study demonstrates that HuGentra may serve as a suitable human ECM substitute for hiPSCs differentiation into the three representative lineage cell types of neurons, cardiomyocytes, and hepatocytes when compared to Matrigel or the lineage preferred substrate. Cells differentiated on HuGentra showed similar morphologic characteristics, marker expression, and gene expression as cells differentiated on Matrigel. a
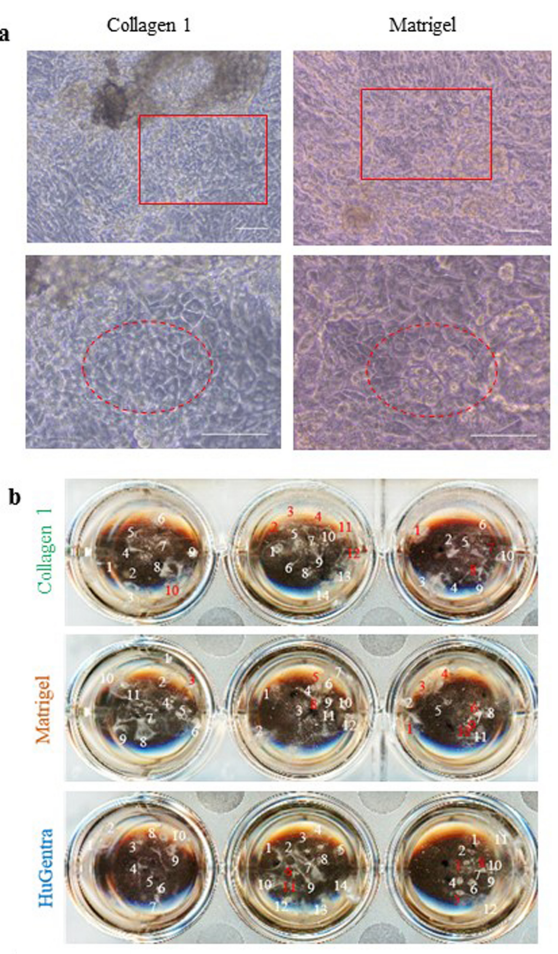

HuGentra

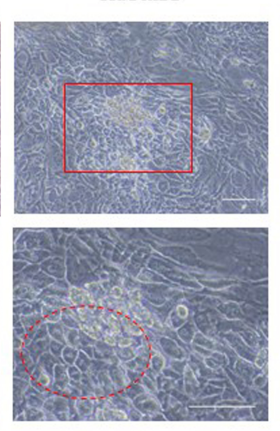

c

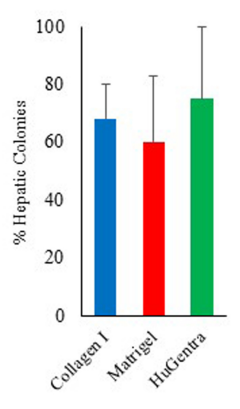

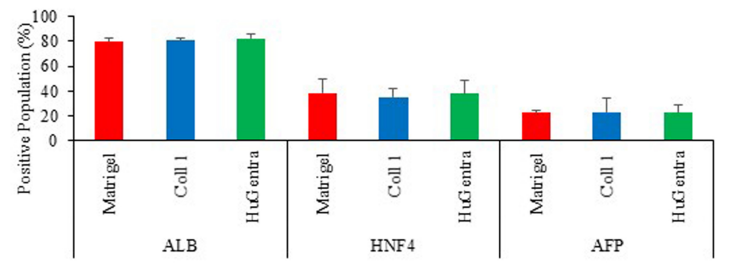

e

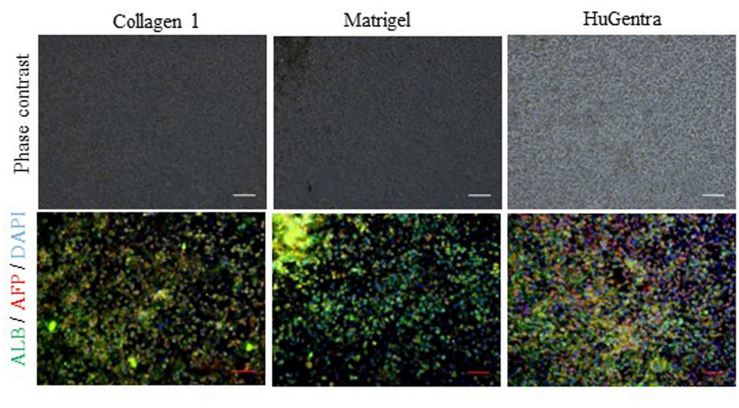

f

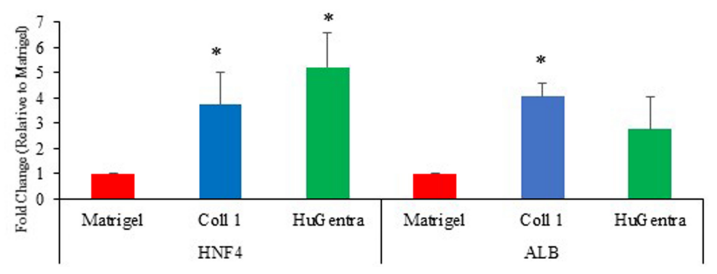

Fig. 4. Characterization of hiPSC-derived hepatocytes on matrices. (a) The frequency of the attached EBs and the resulting colonies were observed and recorded as containing the morphology of hepatic cells. Representative images were taken $10 \times$ (top row) and $20 \times$ (bottom row). (b) A representative plate with labeled hepatic colonies in white and non-hepatic colonies in red. (c) The percentage of EBs containing the characteristic morphology were calculated as a percentage of the total attached EBs. (d) Quantification of ALB, HNF4, and AFP on the dissociated cells via flow cytometry showed no significant differences in expression efficiencies across the three matrices. (e) ICC analysis for ALB and AFP was shown on differentiated hepatic like cells. Representative images were taken $10 \times$. (f) Gene expression of HNF4 and $A L B$ on Collagen I and HuGentra normalized to expression on Matrigel. Error bars represent standard deviation. ${ }^{*} \mathrm{p}<0.05$ to hiPSCs differentiated on Matrigel. $\mathrm{n} \geq 3$. 
HuGentra complements a humanized culture system requiring a growth substrate, enabling the use of hiPSCs for regenerative medicine in the future. Additionally, HuGentra may become a valuable tool in the development of hiPSC derived in vitro screening platforms.

\section{Acknowledgments}

All experiments were funded by LifeNet Health with guidance from Jung Bok Lee.

\section{Potential Conflict of Interest}

The authors are currently or formally employed by Lifenet Health.

\section{References}

1. Kleinman HK, Martin GR. Matrigel: basement membrane matrix with biological activity. Semin Cancer Biol 2005;15: 378-386

2. Del Álamo JC, Lemons D, Serrano R, Savchenko A, Cerignoli F, Bodmer R, Mercola M. High throughput physiological screening of iPSC-derived cardiomyocytes for drug development. Biochim Biophys Acta 2016;1863(7 Pt B): $1717-1727$

3. Kondo Y, Iwao T, Nakamura K, Sasaki T, Takahashi S, Kamada N, Matsubara T, Gonzalez FJ, Akutsu H, Miyagawa Y, Okita H, Kiyokawa N, Toyoda M, Umezawa A, Nagata $\mathrm{K}$, Matsunaga T, Ohmori S. An efficient method for differentiation of human induced pluripotent stem cells into hepatocyte-like cells retaining drug metabolizing activity. Drug Metab Pharmacokinet 2014;29:237-243

4. Lee JB, Graham M, Collins TJ, Lee JH, Hong SH, Mcnicol AJ, Shapovalova Z, Bhatia M. Reversible lineage-specific priming of human embryonic stem cells can be exploited to optimize the yield of differentiated cells. Stem Cells 2015;33:1142-1152

5. Lian X, Hsiao C, Wilson G, Zhu K, Hazeltine LB, Azarin SM, Raval KK, Zhang J, Kamp TJ, Palecek SP. Robust cardiomyocyte differentiation from human pluripotent stem cells via temporal modulation of canonical Wnt signaling. Proc Natl Acad Sci U S A 2012;109:E1848-E1857

6. Tannenbaum SE, Turetsky TT, Singer O, Aizenman E, Kirshberg S, Ilouz N, Gil Y, Berman-Zaken Y, Perlman TS, Geva N, Levy O, Arbell D, Simon A, Ben-Meir A, Shufaro Y, Laufer N, Reubinoff BE. Derivation of xeno-free and GMP-grade human embryonic stem cells--platforms for future clinical applications. PLoS One 2012;7:e35325.

7. Zhao C, Ikeya M. Generation and applications of induced pluripotent stem cell-derived mesenchymal stem cells. Stem Cells Int 2018;2018:9601623

8. Hongisto $\mathrm{H}$, Ilmarinen $\mathrm{T}$, Vattulainen $\mathrm{M}$, Mikhailova A, Skottman H. Xeno- and feeder-free differentiation of human pluripotent stem cells to two distinct ocular epithelial cell types using simple modifications of one method. Stem Cell Res Ther 2017;8:291

9. Farzaneh Z, Pakzad M, Vosough M, Pournasr B, Baharvand H. Differentiation of human embryonic stem cells to hepatocyte-like cells on a new developed xeno-free extracellular matrix. Histochem Cell Biol 2014;142:217-226

10. Ullah I, Busch JF, Rabien A, Ergün B, Stamm C, Knosalla C, Hippenstiel S, Reinke P, Kurtz A. Adult tissue extracellular matrix determines tissue specification of human iPSC-derived embryonic stage mesodermal precursor cells. Adv Sci (Weinh) 2020;7:1901198

11. Francis MP, Breathwaite E, Bulysheva AA, Varghese F, Rodriguez RU, Dutta S, Semenov I, Ogle R, Huber A, Tichy AM, Chen S, Zemlin C. Human placenta hydrogel reduces scarring in a rat model of cardiac ischemia and enhances cardiomyocyte and stem cell cultures. Acta Biomater 2017;52:92-104 